\title{
Screening for rheumatic heart disease: quality and agreement of focused cardiac ultrasound by briefly trained health workers
}

Daniel Engelman ${ }^{1,2,3^{*}}$ D, Joseph H. Kado ${ }^{4,5}$, Bo Reményi ${ }^{6,7}$, Samantha M. Colquhoun ${ }^{1,2,7}$, Jonathan R. Carapetis ${ }^{8,9}$, Nigel J. Wilson ${ }^{10,11}$, Susan Donath ${ }^{12,13}$ and Andrew C. Steer ${ }^{1,2,3}$

\begin{abstract}
Background: Echocardiographic screening for rheumatic heart disease (RHD) has the potential to detect subclinical cases for secondary prevention, but is constrained by inadequate human resources in most settings. Training nonexpert health workers to perform focused cardiac ultrasound (FoCUS) may enable screening at a population-level. We aimed to evaluate the quality and agreement of FoCUS for valvular regurgitation by briefly trained health workers.

Methods: Seven nurses participated in an eight week training program in Fiji. Nurses performed FoCUS on 2018 children aged five to 15 years, and assessed any valvular regurgitation. An experienced pediatric cardiologist assessed the quality of ultrasound images and measured any recorded regurgitation. The assessment of the presence of regurgitation and measurement of the longest jet by the nurse and cardiologist was compared, using the Bland-Altman method.

Results: The quality of FoCUS overall was adequate for diagnosis in $96.6 \%$. There was substantial agreement between the cardiologist and the nurses overall on the presence of mitral regurgitation $(k=0.75)$ and aortic regurgitation $(k=0.61)$ seen in two views. Measurements of mitral regurgitation by nurses and the cardiologist were similar (mean bias $0.01 \mathrm{~cm}$; $95 \%$ limits of agreement -0.64 to $0.66 \mathrm{~cm}$ ).

Conclusions: After brief training, health workers with no prior experience in echocardiography can obtain adequate quality images and make a reliable assessment on the presence and extent of valvular regurgitation. Further evaluation of the imaging performance and accuracy of screening by non-expert operators is warranted, as a potential population-level screening strategy in high prevalence settings.
\end{abstract}

Keywords: Echocardiography, Cardiac ultrasound, Rheumatic heart disease, Mass screening, Task shifting

\section{Background}

Rheumatic heart disease (RHD) remains a major cause of morbidity and mortality in resource-poor countries, with an estimated 30 million prevalent cases globally causing in excess of 345,000 deaths annually [1]. The World Health Organization recommends population-based screening for

\footnotetext{
* Correspondence: Daniel.Engelman@rch.org.au

${ }^{1}$ Centre for International Child Health, University of Melbourne, Parkville, Victoria, Australia

${ }^{2}$ Group A Streptococcal Research, Murdoch Childrens Research Institute, Parkville, Victoria, Australia

Full list of author information is available at the end of the article
}

early case detection in high-prevalence areas [2], but very few countries have implemented screening within RHD control programs. Echocardiography has considerably greater accuracy than clinical examination for the diagnosis of mild or asymptomatic RHD [3, 4], and consensus guidelines developed by the World Heart Federation (WHF) [5] allow a standardised definition of 'definite' or 'borderline' RHD on diagnostic echocardiography. However, the application of these advances in diagnosis to the field of mass screening remains uncertain [6]. 
A major obstacle to RHD screening at a population-scale is the insufficient number of health workers skilled in echocardiography. Standard training in echocardiography requires considerable time and practice $[7,8]$ and may not be feasible. An alternate approach to increase capacity may be to develop shorter, focused training programs and reallocate simplified cardiac ultrasound to non-expert health workers, known as task shifting [9]. Task shifting strategies can extend and strengthen available health services [10,11], yet require operational research prior to introduction to ensure adequate performance and quality [12].

In parallel to considerations of building health workforce capacity, there has been growing acknowledgement of the potential application of focused cardiac ultrasound (FoCUS), rather than standard comprehensive echocardiography, for a range of clinical scenarios, including by briefly trained, non-expert operators [13, 14]. The recent development of international, evidence-based recommendations for FoCUS is therefore highly relevant to RHD screening [15].

In the setting of Fiji, the national capacity to perform echocardiography for RHD is limited to seven physicians and one technologist. Nurses are the largest human resource of the health system (approximately 1 nurse per 500 population) [16], and deliver a range of health services, including school-based health care [11, 17].

In this context, we sought to determine whether shifting FoCUS tasks to the existing school-based nurse workforce was a feasible strategy to implement RHD screening. A pilot study demonstrated that training two nurses to perform basic echocardiography for RHD to facilitate referral was possible, using the identification and measurement of regurgitation jet length as a risk marker of disease [18]. Therefore, we designed a two-part study to further assess this strategy. The first component, presented here, aimed to evaluate the performance of a larger number of nonexpert operators in the identification and measurement of valvular regurgitation, after a defined training program. The specific objectives of this study were to evaluate the quality of FoCUS performed by briefly trained nurses; evaluate the inter-rater agreement between these nurses and an expert cardiologist on the presence of valvular regurgitation; and compare the measurements of regurgitation length made by the nurses and cardiologist.

The second component of the study, an investigation of the accuracy (sensitivity and specificity) of screening by non-expert operators, compared with a reference standard diagnosis of RHD, would only be relevant if the quality and agreement were acceptable.

\section{Methods}

\section{Setting}

The study took place in Fiji, a nation of 332 islands in the South Pacific Ocean with a population of approximately
900,000. The Fiji RHD Control Program commenced in 2005. The burden of RHD is among the highest in the world [19], with a prevalence of definite RHD of 8.4 per 1000 children aged 5-14 [20].

\section{Training}

Seven nurses were enrolled in the training program, representing two nurses from each of the major administrative Divisions of Fiji, and one additional nurse to cover the possibility of dropout. None had any previous experience in echocardiography or cardiology. Training was held in Suva, Fiji in in June-July 2012, and has been previously described in detail [21]. The program consisted of one week of classroom-based workshops, run by a pediatric cardiologist and a pediatrician experienced in echocardiography for RHD. This was followed by seven weeks of practical training, where nurses had the opportunity to practice with volunteer children in a school-setting, supervised by an experienced technician. There was no summative or "hurdle" assessment of competency, and as the study aimed to assess this defined training program, no review of images or ongoing training was provided once nurses commenced ultrasound assessment.

\section{Nurse assessment of regurgitation}

Nurses performed FoCUS assessments from September 2012 to September 2013 in eight schools, encompassing all three Divisions (Fig. 1). Nurses performed a simplified 12-step protocol focussing on the left side of the heart (Table 1). They were instructed to record the presence of any mitral regurgitation (MR) or aortic regurgitation (AR) on color Doppler imaging in the parasternal long axis, parasternal short axis and apical views, and if present, to measure the longest visible jet in the parasternal long axis and/or apical views. Nurses made assessments and measurements at the time of examination. To minimise the measurement of closing volumes, nurses were instructed to only measure regurgitation seen in $\geq 2$ frames. Nurses were not expected to identify morphological changes of the valves.

The nurses used the M-Turbo portable ultrasound machine (SonoSite Inc., Bothell, WA) which is relatively affordable whilst maintaining adequate quality for assessment of regurgitation on color Doppler. Spectral Doppler is also available but was not used in this study. The machine can run on mains power or battery for several hours, which was a practical advantage over hand-held ultrasound equipment. A 5-1 $\mathrm{MHz}$ transducer was used for all studies. Nurses were provided with a simplified manual and taught to operate and adjust relevant settings on the machines. Depth and gain were manually adjusted for each examination. Nyquist limits were set to maximum to 


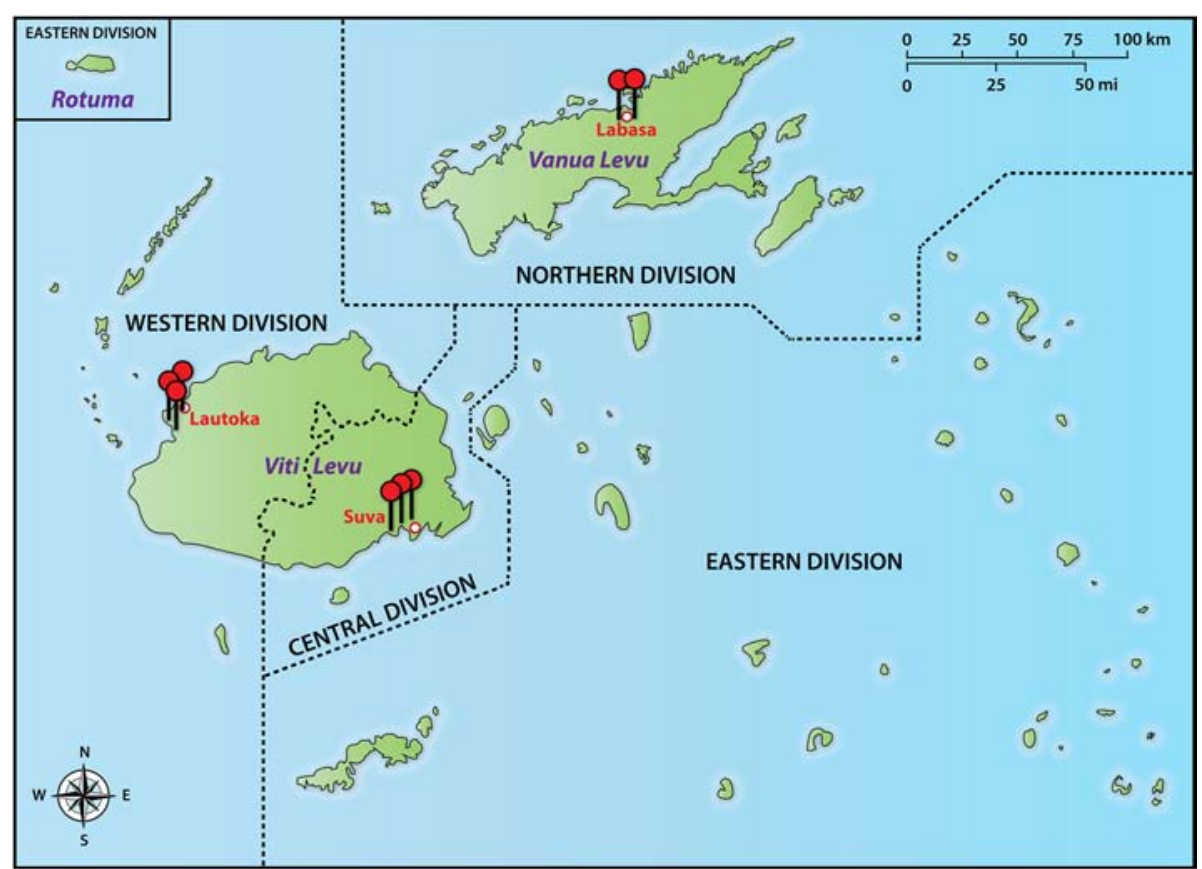

Fig. 1 Map of study sites in Fiji. Pins indicate school assessment sites

avoid overestimation of jet length. All images and loops were saved. Nurses recorded the time examinations commenced (prior to participant lying down) and finished (after saving images and completing data forms). Nurse assessment data were entered into an EpiData electronic database (version 3.1, The EpiData Association, Odense, Denmark).

Table 1 Twelve step focused cardiac ultrasound protocol for assessment of regurgitation

\begin{tabular}{lllll}
\hline View & Valve & Mode & $\begin{array}{l}\text { Identify } \\
\text { regurgitation }\end{array}$ & $\begin{array}{l}\text { Measure } \\
\text { longest jet }\end{array}$ \\
\hline 1. PLAX & Mitral & 2D & & Yes \\
& Mitral & CD & Yes & Yes \\
& Aortic & 2D & & \\
2. PSAX & Aortic & CD & Yes & No \\
& Aortic & 2D & & No \\
& Aortic & CD & Yes & \\
& Mitral & 2D & & Yes \\
& Mitral & CD & Yes & \\
& Mitral & 2D & & Yes \\
& Mitral & CD & Yes & \\
& Aortic & 2D & & Yes \\
& Aortic & CD & &
\end{tabular}

PLAX parasternal long-axis view, $P S A X$, parasternal short-axis view, $2 D$ two-dimensional imaging mode, $C D$, color Doppler mode

\section{Cardiologist reporting}

De-identified ultrasounds were sent securely to a pediatric cardiologist for reporting. The cardiologist reported the quality of each echocardiographic view on a scale adapted from similar studies [22] as one of: (1) adequate; (2) poor quality but assessment made; or (3) not interpretable. The categorization was based on an assessment of the completeness of images and loops recorded, orientation, axis and clarity of anatomical structures. Regurgitation present for $\geq 2$ frames in any view was noted, and measured if present in the parasternal long axis and/or apical views (i.e., the same assessment as done by the nurse operators). Reports were entered into a REDCap electronic database hosted at the Murdoch Childrens Research Institute [23].

\section{Participants}

Schools were selected from each Division so that each nurse would perform approximately equal numbers of FoCUS examinations. The Fiji Ministry of Education granted permission for research staff to visit each school to explain the procedure to the students, parents, and teachers. Information sheets in Fijian and English were provided to the families of participants. All children aged five to 15 years attending the selected schools were eligible to participate. Signed consent was obtained from the parent or guardian, and assent was additionally required for children aged 10 years and above. Participants also had a standard echocardiogram performed by a 
sonographer, and were referred to a pediatrician if any abnormalities were suspected. As the seven nurses were also subjects of this study, they gave informed consent prior to participation.

\section{Statistical analysis}

De-identified data were analysed using Stata (version 12, Stata Corp, Texas, USA). The proportion of examinations in each quality category were compared overall, and for each echocardiographic view. Cohen's kappa (к) statistic was used to evaluate the inter-rater agreement for the nurses' and cardiologist's assessment of the presence of regurgitation in each view, and for regurgitation jet lengths at $0.5 \mathrm{~cm}$ intervals up to the WHF diagnostic cut-off point ( $2 \mathrm{~cm}$ for MR, $1 \mathrm{~cm}$ for AR). The regurgitation jet length measurements by the nurses and cardiologist were compared using the Bland-Altman method [24]; the difference between length measurements was plotted against the average length measurement, and the $95 \%$ limits of agreement were calculated (mean bias \pm 1.96 times standard deviations of the bias).

\section{Sample size}

Since the study aim was to compare the measurement of regurgitation length between nurses and cardiologist, the sample size was calculated to ensure a minimum degree of precision for the estimated results. With a sample size of 100 paired measurements, the $95 \%$ confidence interval for each of the Bland-Altman $95 \%$ limits of agreement has a width of 0.34 times the standard deviation of the paired differences [24]. The confidence interval for the overall limits of agreement would therefore be around $\sim 4 \%$ wider than the difference in limits; we considered this to be an adequate degree of precision. We estimated that paired measurements of regurgitation would be available for $15 \%$ (MR) and $5 \%$ (AR) of the sample, and therefore aimed to recruit 2000 children.

\section{Ethical approval}

The study was approved by the Fiji National Health Research Committee, Fiji (Ref 2011 051) and the Human Research Ethics Committee of Northern Territory Department of Health and Menzies School of Health Research, Australia (Ref 11-1649).

\section{Results}

Two thousand eighteen children were enrolled. The number of children assessed by the seven nurses ranged from 239 (11.8 \%) to 340 (16.9\%). The mean age was 10.1 years (range 5.1 - 15.7 years) and $51.3 \%$ were female. The ethnicity of participants was $60.9 \%$ iTaukei (indigenous Fijian), $36.6 \%$ Fijians of Indian descent and $2.5 \%$ other ethnicities.

\section{Quality}

The overall quality of nurse FoCUS was adequate in 1949 (96.6 \%) examinations; poor but interpretable in 58 $(2.9 \%)$ and not interpretable in 11 (0.6 \%, Table 2). One nurse had an overall quality (adequate: $81.8 \%$ ) that was much lower compared to the other six nurses (adequate: range 97.1 - 99.4\%), and most (48 of 58) of the poor and not interpretable examinations were performed by this nurse.

\section{Agreement of regurgitation assessment}

There was substantial agreement between the cardiologist and the nurses overall on the presence of MR ( $\mathrm{K}=$ $0.75)$ and AR ( $\mathrm{K}=0.61)$ seen in two of the three echocardiographic views. The agreement increased when combined with minimum jet length criteria, such that the agreement was highest for $\mathrm{MR} \geq 1.5 \mathrm{~cm}(\kappa=0.81)$ and $\geq 2.0 \mathrm{~cm}(\mathrm{~K}=0.78)$; and for $\mathrm{AR} \geq 0.5 \mathrm{~cm}(\mathrm{~K}=0.64)$ and $\geq 1.0 \mathrm{~cm}(\mathrm{\kappa}=0.65)$. There was moderate agreement on the presence of MR or AR in any one view, and moderate to substantial agreement for individual single views (Table 3). Details of assessment and agreement are shown in Additional file 1: Table S1.

\section{Comparison of measurements}

There were 442 paired measurements of mitral regurgitation in either the parasternal long axis or apical views, with a mean bias of $0.01 \mathrm{~cm}$ and $95 \%$ limits of agreement -0.64 to $0.66 \mathrm{~cm}$ (Fig. 2). The number of paired measures for aortic regurgitation $(\mathrm{n}=36)$ was insufficient to calculate the $95 \%$ limits of agreement with confidence, however the mean bias was small $(-0.12 \mathrm{~cm})$, most measures were very similar and there were few differences greater than $1 \mathrm{~cm}$. The comparison of measurements in the parasternal long axis and apical views measurements were similar (Additional file 2: Figure S1). In all comparisons, the mean and standard deviation appear uniform through the range of measurements.

\section{Screening logistics}

The median duration for each ultrasound performed by the nurses was $12 \mathrm{~min}$, (interquartile range (IQR), 9 $17 \mathrm{~min}$ ) inclusive of saving images and completing all research forms. Each nurse tested a median 11.7 children per day (maximum 30, IQR 8-14).

\section{Discussion}

Our study shows that after completing a brief, structured training program, seven nurses acquired adequate quality FoCUS images in a high proportion of cases. There was moderate to substantial agreement on the presence of regurgitation between nurses and the reporting cardiologist, and measurements of regurgitation jet lengths were similar. 
Table 2 Quality of focused cardiac ultrasound by nurses

\begin{tabular}{|c|c|c|c|c|c|c|c|c|c|c|c|c|c|c|c|c|c|}
\hline \multirow[t]{2}{*}{ View } & \multirow[t]{2}{*}{ Quality } & \multicolumn{2}{|c|}{$\begin{array}{l}\text { Nurse } 1 \\
n=250\end{array}$} & \multicolumn{2}{|c|}{$\begin{array}{l}\text { Nurse } 2 \\
n=264\end{array}$} & \multicolumn{2}{|c|}{$\begin{array}{l}\text { Nurse } 3 \\
n=340\end{array}$} & \multicolumn{2}{|c|}{$\begin{array}{l}\text { Nurse } 4 \\
n=340\end{array}$} & \multicolumn{2}{|c|}{$\begin{array}{l}\text { Nurse } 5 \\
n=274\end{array}$} & \multicolumn{2}{|c|}{$\begin{array}{l}\text { Nurse } 6 \\
n=311\end{array}$} & \multicolumn{2}{|c|}{$\begin{array}{l}\text { Nurse } 7 \\
n=239\end{array}$} & \multicolumn{2}{|c|}{$\begin{array}{l}\text { Total } \\
\mathrm{n}=2018\end{array}$} \\
\hline & & $\mathrm{n}$ & $\%$ & $\mathrm{n}$ & $\%$ & $\mathrm{n}$ & $\%$ & $\mathrm{n}$ & $\%$ & $\mathrm{n}$ & $\%$ & $\mathrm{n}$ & $\%$ & $\mathrm{n}$ & $\%$ & $\mathrm{n}$ & $\%$ \\
\hline \multirow[t]{3}{*}{ Overall } & 1 & 248 & 99.2 & 216 & 81.8 & 338 & 99.4 & 337 & 99.1 & 266 & 97.1 & 308 & 99.0 & 236 & 98.7 & 1949 & 96.6 \\
\hline & 2 & 2 & 0.8 & 42 & 15.9 & 0 & 0.0 & 2 & 0.6 & 7 & 2.6 & 3 & 1.0 & 2 & 0.8 & 58 & 2.9 \\
\hline & 3 & 0 & 0.0 & 6 & 2.3 & 2 & 0.6 & 1 & 0.3 & 1 & 0.4 & 0 & 0.0 & 1 & 0.4 & 11 & 0.5 \\
\hline \multirow[t]{3}{*}{ MV 2D } & 1 & 247 & 98.8 & 204 & 77.3 & 337 & 99.1 & 337 & 99.1 & 265 & 96.7 & 308 & 99.0 & 235 & 98.3 & 1933 & 95.8 \\
\hline & 2 & 3 & 1.2 & 59 & 22.3 & 1 & 0.3 & 3 & 0.9 & 9 & 3.3 & 3 & 1.0 & 4 & 1.7 & 82 & 4.0 \\
\hline & 3 & 0 & 0.0 & 1 & 0.4 & 2 & 0.6 & 0 & 0.0 & 0 & 0.0 & 0 & 0.0 & 0 & 0.0 & 3 & 0.2 \\
\hline \multirow[t]{3}{*}{ MV CD } & 1 & 249 & 99.6 & 210 & 79.5 & 337 & 99.1 & 334 & 98.2 & 256 & 93.4 & 308 & 97.1 & 228 & 95.4 & 1916 & 94.9 \\
\hline & 2 & 1 & 0.4 & 48 & 18.2 & 1 & 0.3 & 5 & 1.5 & 17 & 6.2 & 3 & 2.9 & 10 & 4.2 & 91 & 4.5 \\
\hline & 3 & 0 & 0.0 & 6 & 2.3 & 2 & 0.6 & 1 & 0.3 & 1 & 0.4 & 0 & 0.0 & 1 & 0.4 & 11 & 0.5 \\
\hline \multirow[t]{3}{*}{ AV 2D } & 1 & 244 & 97.6 & 215 & 81.4 & 329 & 96.7 & 325 & 95.6 & 268 & 97.8 & 302 & 99.0 & 236 & 98.7 & 1925 & 95.4 \\
\hline & 2 & 6 & 2.4 & 49 & 18.6 & 9 & 2.7 & 15 & 4.4 & 5 & 1.8 & 9 & 1.0 & 3 & 1.3 & 90 & 4.5 \\
\hline & 3 & 0 & 0.0 & 0 & 0.0 & 2 & 0.6 & 0 & 0.0 & 1 & 0.4 & 0 & 0.0 & 0 & 0.0 & 3 & 0.1 \\
\hline \multirow[t]{3}{*}{ AV CD } & 1 & 248 & 99.2 & 224 & 84.8 & 338 & 99.4 & 340 & 100.0 & 266 & 97.1 & 308 & 99.4 & 236 & 98.3 & 1960 & 97.1 \\
\hline & 2 & 2 & 0.8 & 38 & 14.4 & 0 & 0.0 & 0 & 0.0 & 7 & 2.6 & 3 & 0.6 & 3 & 1.7 & 53 & 2.6 \\
\hline & 3 & 0 & 0.0 & 2 & 0.8 & 2 & 0.6 & 0 & 0.0 & 1 & 0.4 & 0 & 0.0 & 0 & 0.0 & 5 & 0.3 \\
\hline
\end{tabular}

Quality: 1, adequate for diagnosis; 2, poor but diagnosis made; 3, not interpretable. $A V$ aortic valve, $M V$ mitral valve, $2 D$ two-dimensional mode, $C D$ color Doppler mode

The proportion of ultrasound studies that were considered adequate quality for diagnosis was high, with very few studies $(<1 \%)$ deemed inadequate for remote diagnosis. However, the images of one nurse were below the necessary standard, with approximately $20 \%$ inadequate. This variability emphasises the importance of ensuring competency in imaging technique [15], and ongoing quality assurance and monitoring as part of screening program design [25]. As our study was designed to evaluate a pre-defined training program, we intentionally did not incorporate interim review of field

Table 3 Agreement between nurse and cardiologist on the presence of regurgitation

\begin{tabular}{llll}
\hline View & Jet length $(\mathrm{cm})$ & $\begin{array}{l}\text { Mitral } \\
\text { kappa }(\mathrm{Cl})\end{array}$ & $\begin{array}{l}\text { Aortic } \\
\text { kappa }(\mathrm{Cl})\end{array}$ \\
\hline One view & $>0$ & $0.60(0.56,0.64)$ & $0.44(0.32,0.57)$ \\
PLAX & $>0$ & $0.72(0.70,0.74)$ & $0.54(0.42,0.62)$ \\
PSAX & $>0$ & $0.55(0.53,0.57)$ & $0.53(0.45,0.61)$ \\
Apical & $>0$ & $0.61(0.58,0.64)$ & $0.44(0.33,0.58)$ \\
Two views & $>0$ & $0.75(0.71,0.80)$ & $0.61(0.46,0.77)$ \\
Two views & 0.5 & $0.76(0.71,0.80)$ & $0.64(0.49,0.79)$ \\
Two views & 1.0 & $0.78(0.74,0.83)$ & $0.65(0.49,0.81)$ \\
Two views & 1.5 & $0.81(0.76,0.86)$ & $\mathrm{n} / \mathrm{a}^{\mathrm{a}}$ \\
Two views & 2.0 & $0.78(0.71,0.86)$ & $\mathrm{n} / \mathrm{a}^{\mathrm{a}}$
\end{tabular}

Cl $95 \%$ confidence interval, PLAX parasternal long axis, PSAX parasternal short axis. Jet length refers to minimum jet length in at least one view. ${ }^{\text {a Analysis }}$ was not performed for jet lengths greater than the World Heart Federation diagnostic cut-off point images or progressive training. However, we recommend that if non-expert operators are employed for programmatic screening, regular review and ongoing training should be incorporated, which may detect and address inadequacies in specific screening competencies, or with individual operators. Future training procedures could also include a requirement for operators to achieve a minimum standard for image quality. The additional human resource requirements for this ongoing training, supervision and quality assurance should be incorporated into screening program planning. Some variability of imaging performance is to be expected, as the study involved seven individuals. This highlights the inherent variability of workers to perform specific tasks, and the importance of training the most suitable staff for each task.

There was moderate to substantial agreement on the presence of valvular regurgitation. These results are more notable given the conditions: nurses made rapid assessments and measurements at the time of imaging in the field, whereas the cardiologist reported under optimal conditions, including time and the ability to scroll though images and individual frames. Agreement was higher when assessing regurgitation present in two views, which aligns with the definition of pathological regurgitation used in several iterations of RHD diagnostic criteria [5, 26]. Agreement was highest for regurgitation with a minimum jet length at, or $0.5 \mathrm{~cm}$ shorter than, the WHF diagnostic cut-off point. These jets are 

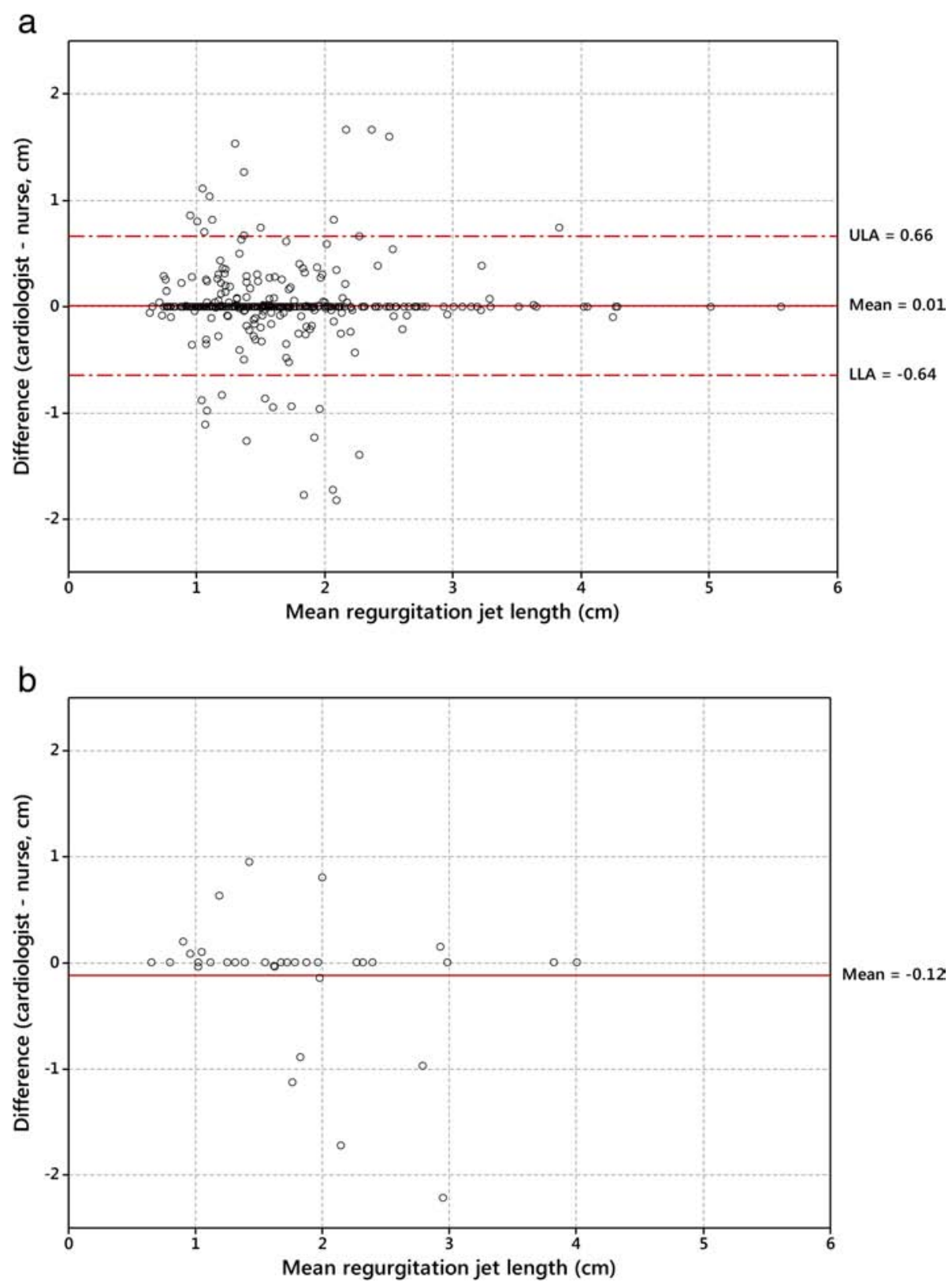

Fig. 2 Difference versus mean (Bland-Altman) plots comparing cardiologist and nurse measurements of regurgitation length. a: Mitral regurgitation; b: Aortic regurgitation. ULA and LLA indicate upper and lower $95 \%$ limits of agreement

more likely to be clinically significant. The agreement between nurses and cardiologist in our study compare favourably to the agreement between experienced cardiologists (kappa $0.4-0.6$ ) on the presence of significant regurgitation reported by Roberts et al. in Australia [27].

Despite the overall high agreement, the cases of disagreement warrant further consideration. A large proportion of these jets were short and clinically insignificant, representing physiological regurgitation or closing volumes (Fig. 3). This may be related to the Doppler mode of the ultrasound machine used in the study, which we found to be highly sensitive for detecting closing volumes, usually visible for only one frame and never pansystolic or pandiastolic. Our protocol attempted to allow for this by reporting only multiple-frame jets, although it is possible that the protocol was not followed in all cases, or that some closing volumes were visible in multiple frames. Disagreements for MR included cases where either the cardiologist or the nurse identified a jet, but disagreements for AR appeared to solely represent jets identified by the cardiologist but missed by the nurses. This may indicate the need for further training for the less common finding of AR. 

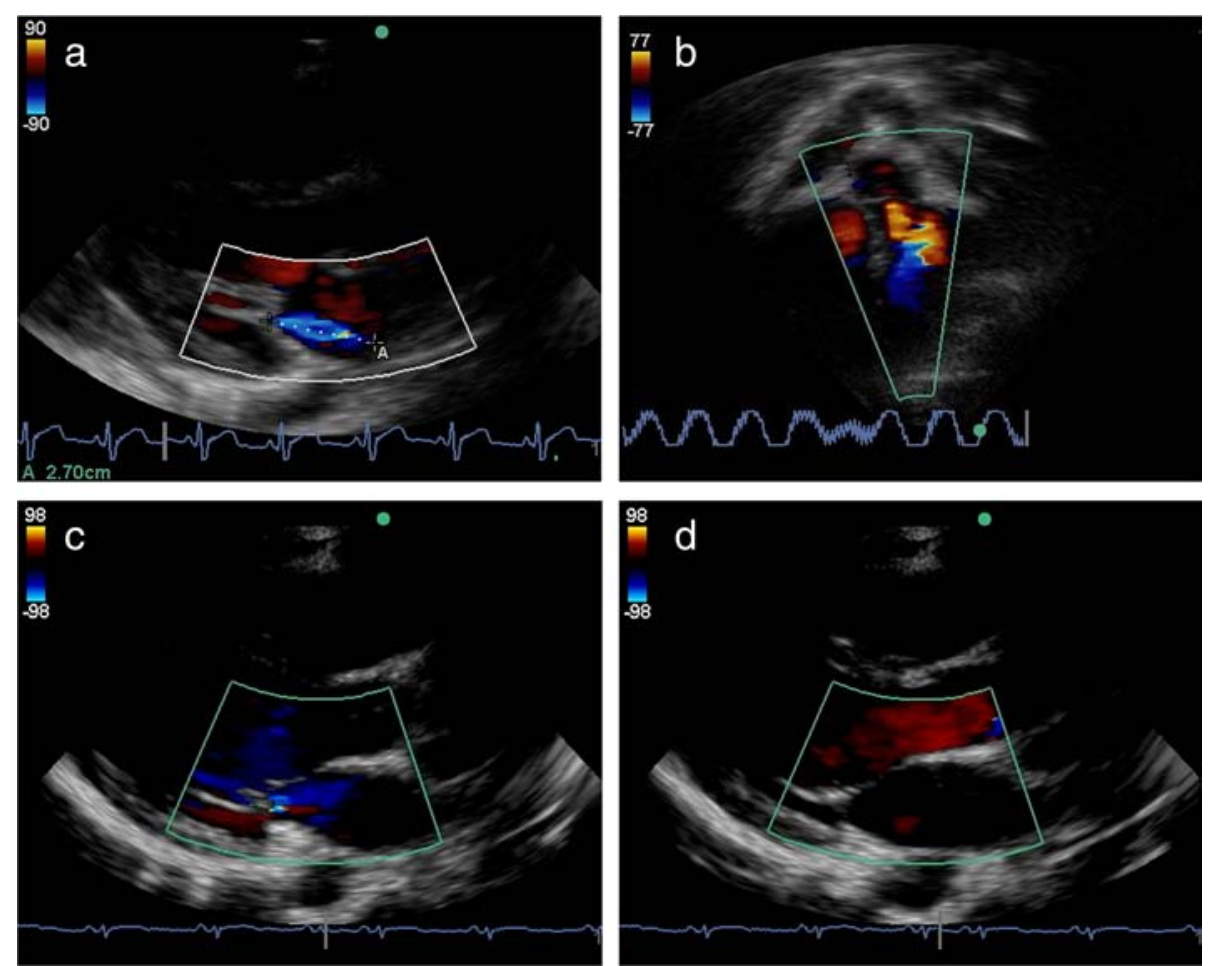

Fig. 3 Examples of cardiac ultrasound performed by nurses. a. Parasternal long axis view demonstrating mitral regurgitation. This image was categorised adequate quality. $\mathbf{b}$. Apical 5 chamber view demonstrating aortic regurgitation. The regurgitation was not identified by the nurse. This image was categorised poor quality. $\mathbf{c}$ and $\mathbf{d}$. Parasternal long axis view demonstrating closing volume. Color is seen across the mitral valve in single frame at the time of valve closure, but not in mid-systole

The measurements of regurgitation made by the nurses and the cardiologist were similar in most cases, but there were several large discrepancies. Based on our results, regurgitation length measurements would be expected to be within $1.3 \mathrm{~cm}$ for $95 \%$ of individuals assessed. The clinical significance of this result at a population-level is uncertain, however, any discrepancy around the regurgitation length cut-off point may affect the classification between normal or pathological regurgitation in some cases. Overall these findings are encouraging, suggesting non-expert operators with limited training, can not only detect, but also measure regurgitation with adequate reliability in the field. Further studies examining the comparison of measurements, for example, between cardiologists, may help establish the clinical significance of these measurement comparison results.

Our training and evaluation concentrated on valvular regurgitation as a potential risk marker for RHD screening. Four recent publications, using different methods, have also proposed utilising regurgitation as a risk marker [28-31]. However, there are inherent limitations of this approach. Physiological mitral regurgitation is a common and benign finding on echocardiography [32, 33], and therefore measuring regurgitation alone may not differentiate between healthy and diseased individuals. Diagnostic criteria for RHD endeavour to differentiate normal from disease by specifying velocity and spectral envelope requirements for pathological regurgitation and additionally requiring valve morphology abnormalities [5]. However, our experience is that morphological assessment of valve appearance and continuous-wave Doppler are advanced skills beyond the capabilities of non-expert operators (although it may be possible for some workers to acquire these skills with further training and experience.) Further, a screening test using only regurgitation would not differentiate between congenital and rheumatic causes of pathological regurgitation [33], and may miss cases of mitral stenosis and potentially treatable congenital lesions [34]. These factors support the need for diagnostic echocardiography and clinical assessment to determine the final diagnosis for those with abnormal screening tests.

Our study has some limitations. The cardiologist was able to only report on the images saved, and could not determine if a nurse had failed to record a regurgitant jet. This may have resulted in an overestimation of agreement. As each child was assessed by one nurse, and all images reported by a single cardiologist, the assessment of inter-rater agreement between nurses, or between reporting cardiologists was not possible. In 
addition, there were insufficient cases of AR to accurately calculate the limits of agreement.

The results are representative only of the conditions of our study: the specific training program, the ultrasound machine and protocols, the seven participating nurses and the Fiji school-aged population. Therefore, our findings should be translated with caution to other settings, or using different training programs. The identification and measurement of jets on color Doppler imaging is related to equipment characteristics, and further evaluation using alternate portable or hand-held ultrasound systems is required. In one such recent evaluation, Mirabel and colleagues reported their experience training two nurses using hand-held machines in New Caledonia [30]. After a three-day theoretical component and more than $50 \mathrm{~h}$ of highly-supervised practical training, the two nurses obtained good (37-43 \%) or fair (56-59 \%) images for most patients, and had high sensitivity and specificity (approximately 80 and $90 \%$ ) for the detection of RHD compared with a cardiologist assessment. The intensive one-to-one or two-to-one, tailored tutorship used in that study may be more effective for some aspects of imaging, but less feasible in resource-poor settings. Ploutz and colleagues also report high accuracy (sensitivity $74 \%$, specificity $79 \%$ ) of a hand-held screening approach by two nurse operators with some previous echocardiography experience in Uganda [31]. Whilst the results are not directly comparable with our study, both studies support and expand the concept that non-expert operators can learn the skills to perform FoCUS with brief training.

Finally, our research evaluated a human resource strategy to facilitate mass screening in a resource-limited setting, but there are many other important issues pertaining to screening. These include the target population, health system capacity for confirmatory diagnosis and management, the significance of subclinical disease, delivery of effective secondary prophylaxis and costeffectiveness [35, 36].

\section{Conclusion}

We conclude that after an eight-week training program, nurses were able to obtain adequate quality FoCUS images and reliably assess children in a school setting for the presence and extent of valvular regurgitation. Given these findings, we believe an assessment of the diagnostic accuracy (sensitivity, specificity and predictive values) of screening by non-expert operators, compared to the reference standard (diagnosis by an expert cardiologist) is warranted. Further investigation of the imaging performance of non-expert operators, with variations to the training program, ultrasound equipment and setting is also required, and will inform the overall evaluation of the role of population-based screening in the prevention of RHD.

\section{Additional files}

Additional file 1: Table S1. Details of identification of regurgitation by nurse and cardiologist on nurse ultrasound. (DOC $33 \mathrm{~kb}$ )

Additional file 2: Figure S1. Difference versus mean (Bland-Altman) plots comparing cardiologist and nurse measurements of regurgitation length in each view of nurse focused cardiac ultrasound. a: Mitral regurgitation in parasternal long axis view; b: Mitral regurgitation in apical 4-chamber view, c: Aortic regurgitation in parasternal long axis view, d: Aortic regurgitation in apical 5-chamber view. ULA and LLA indicate upper and lower $95 \%$ limits of agreement. (JPG 4469 kb)

\section{Abbreviations}

AR: aortic regurgitation; FoCUS: focused cardiac ultrasound; IQR: interquartile range; MR: mitral regurgitation; RHD: rheumatic heart disease; WHF: World Heart Federation.

\section{Competing interests}

The authors declare that they have no competing interests.

\section{Authors' contributions}

ACS, JHK, and SMC conceived the study, which was designed with DE, BR and JRC. ACS, DE, JHK and BR designed and delivered the training program. DE, SD and ACS designed the statistical methods and data analysis plan. BR and NJW reported the ultrasounds. DE performed the statistical analysis and was the primary author of the manuscript. All authors contributed to the writing of the manuscript, and read and approved the final version.

\section{Acknowledgements}

The Fiji Ministry of Health and Medical Services and Fiji Ministry of Education supported and facilitated this research. We gratefully acknowledge the contribution of research staff Laisiana Matatolu, Frances Matanatabu, Simione Rokosemudu, Sera Rayasidamu. Carol Fox assisted with training. Caroline Watson provided the study map figure. Staff from Clinical Epidemiology and Biostatistics, Murdoch Childrens Research Institute, assisted with data management. We thank the participating nurses, students and schools. Funding for this project was supported by a grant from Cure Kids New Zealand. DE and ACS are both supported by Australian National Health and Medical Research Council / National Heart Foundation research fellowships. DE is also supported by the University of Melbourne Nossal Global Scholars program. SMC is supported by the Australian National Health and Medical Research Council. None of the funders played a role in the design of the study; in the collection, analysis and interpretation of data; in the writing of the report; or in the decision to submit the paper for publication.

\section{Author details}

${ }^{1}$ Centre for International Child Health, University of Melbourne, Parkville, Victoria, Australia. ${ }^{2}$ Group A Streptococcal Research, Murdoch Childrens Research Institute, Parkville, Victoria, Australia. ${ }^{3}$ Royal Children's Hospital, Parkville, Victoria, Australia. ${ }^{4}$ Department of Paediatrics, Colonial War Memorial Hospital, Suva, Fiji. ${ }^{5}$ College of Medicine, Nursing and Health Sciences, Fiji National University, Suva, Fiji. ${ }^{6}$ Royal Darwin Hospital, Tiwi, Northern Territory, Australia. ${ }^{7}$ Menzies School of Health Research, Casuarina, Northern Territory, Australia. ${ }^{8}$ Telethon Kids Institute, University of Western Australia, West Perth, Western Australia, Australia. ${ }^{9}$ Princess Margaret Hospital for Children, Subiaco, Western Australia, Australia. ${ }^{10}$ Starship Hospital, Auckland, New Zealand. ${ }^{11}$ University of Auckland, Auckland, New Zealand. ${ }^{12}$ Department of Paediatrics, University of Melbourne, Parkville, Victoria, Australia. ${ }^{13}$ Clinical Epidemiology and Biostatistics Unit, Murdoch Childrens Research Institute, Parkville, Victoria, Australia.

Received: 31 August 2015 Accepted: 27 January 2016

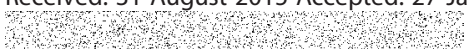

\section{References}

1. Lozano R, Naghavi M, Foreman K, Lim S, Shibuya K, Aboyans V, et al. Global and regional mortality from 235 causes of death for 20 age groups in 1990 and 2010: a systematic analysis for the Global Burden of Disease Study 2010. Lancet. 2012;380:2095-128. 
2. WHO Expert Consultation on Rheumatic Fever and Rheumatic Heart Disease. Rheumatic fever and rheumatic heart disease: report of a WHO Expert Consultation, Geneva, 29 October - 1 November 2001. Geneva: WHO; 2004.

3. Marijon E, Ou P, Celermajer DS, Ferreira B, Mocumbi AO, Jani D, et al. Prevalence of rheumatic heart disease detected by echocardiographic screening. N Engl J Med. 2007;357:470-6.

4. Roberts KV, Brown AD, Maguire GP, Atkinson DN, Carapetis JR. Utility of auscultatory screening for detecting rheumatic heart disease in high-risk children in Australia's Northern Territory. Med J Aust. 2013;199:196-9.

5. Remenyi B, Wilson N, Steer A, Ferreira B, Kado J, Kumar K, et al. World Heart Federation criteria for echocardiographic diagnosis of rheumatic heart disease-an evidence-based guideline. Nat Rev Cardiol. 2012;9:297-309.

6. Zuhlke L, Mayosi BM. Echocardiographic screening for subclinical rheumatic heart disease remains a research tool pending studies of impact on prognosis. Curr Cardiol Rep. 2013;15:343.

7. Popescu BA, Andrade MJ, Badano LP, Fox KF, Flachskampf FA, Lancellotti P, et al. European Association of Echocardiography recommendations for training, competence, and quality improvement in echocardiography. Eur J Echocardiogr. 2009;10:893-905.

8. Ehler D, Carney DK, Dempsey AL, Rigling R, Kraft C, Witt SA, et al. Guidelines for cardiac sonographer education: recommendations of the American Society of Echocardiography Sonographer Training and Education Committee. J Am Soc Echocardiogr. 2001;14:77-84

9. World Health Organization. Transformative scale up of health professional education. Geneva: WHO; 2011.

10. Alliance GHW. Scaling up, saving lives: Task force for scaling up education and training for health workers. Geneva: WHO; 2008.

11. Campbell J, Dussault G, Buchan J, Pozo-Martin F, Guerra Arias M, Leone C, et al. A universal truth: no health without a workforce, Forum Report, Third Global Forum on Human Resources for Health, Recife, Brazil. Geneva: Global Health Workforce Alliance and World Health Organization; 2013.

12. World Health Organization. Task Shifting: rational redistribution of tasks amongst health workforce teams: global recommendations and guidelines. Geneva: WHO; 2008.

13. Shmueli $H$, Burstein $Y$, Sagy I, Perry $Z H$, llia R, Henkin $Y$, et al. Briefly trained medical students can effectively identify rheumatic mitral valve injury using a hand-carried ultrasound. Echocardiography. 2013;30:621-6.

14. Galderisi M, Santoro A, Versiero M, Lomoriello VS, Esposito R, Raia R, et al. Improved cardiovascular diagnostic accuracy by pocket size imaging device in non-cardiologic outpatients: the NaUSiCa (Naples Ultrasound Stethoscope in Cardiology) study. Cardiovasc Ultrasound. 2010;8:51.

15. Via G, Hussain A, Wells M, Reardon R, ElBarbary M, Noble VE, et al. International evidence-based recommendations for focused cardiac ultrasound. J Am Soc Echocardiogr. 2014;27:683. e681-683.e633.

16. Dawson A, Howes T, Gray N, Kennedy E. Human resources for health in maternal, neonatal and reproductive health at community level: A profile of Fiji. Human Resources for Health Knowledge Hub and Burnet Institute: Sydney; 2011.

17. Asia Pacific Observatory on Health Systems and Policies. The Fiji islands health systems review, Health Systems in Transition, vol. 1. Geneva: WHO; 2011.

18. Colquhoun SM, Carapetis JR, Kado JH, Reeves BM, Remenyi B, May W, et al. Pilot study of nurse-led rheumatic heart disease echocardiography screening in Fiji-a novel approach in a resource-poor setting. Cardiol Young. 2013;23:546-52.

19. Carapetis JR, Steer AC, Mulholland EK, Weber M. The global burden of group A streptococcal diseases. Lancet Infect Dis. 2005;5:685-94.

20. Colquhoun SM, Kado JH, Remenyi B, Wilson NJ, Carapetis JR, Steer AC. Echocardiographic screening in a resource poor setting: Borderline rheumatic heart disease could be a normal variant. Int J Cardiol. 2014;173:284-9.

21. Engelman D, Kado JH, Remenyi B, Colquhoun SM, Watson C, Rayasidamu SR, et al. Teaching focused echocardiography for rheumatic heart disease screening. Ann Pediatr Cardiol. 2015;8:118-21.

22. Quiles J, Garcia-Fernandez MA, Avanzas P, Martinez-Selles M, Rosas R, Sanchez Hernandez A, et al. Comparison of echocardiographic studies made with new portable devices to conventional studies. Rev Esp Cardiol. 2003;56:480-6.

23. Harris PA, Taylor R, Thielke R, Payne J, Gonzalez N, Conde JG. Research electronic data capture (REDCap)-a metadata-driven methodology and workflow process for providing translational research informatics support. J Biomed Inform. 2009:42:377-81.

24. Bland JM, Altman DG. Statistical methods for assessing agreement between two methods of clinical measurement. Lancet. 1986;1:307-10.
25. Council of Europe Committee of Ministers. Recommendation No. R (94) 11 of the Committee of Ministers to member states on screening as a tool of preventive medicine. 1994

26. Carapetis JR, Paar J, Cherian T. Standardization of epidemiologic protocols for surveillance of post-streptococcal sequelae: acute rheumatic fever, rheumatic heart disease and acute post-streptococcal glomerulonephritis. National Institutes Health. 2006. http://www.niaid.nih.gov/topics/ strepThroat/Documents/groupasequelae.pdf. Accessed 30 May 2014

27. Roberts K, Maguire G, Brown A, Atkinson D, Remenyi B, Wheaton G, et al. Echocardiographic screening for rheumatic heart disease in high and low risk Australian children. Circulation. 2014;129:1953-61.

28. Mirabel M, Celermajer DS, Ferreira B, Tafflet M, Perier MC, Karam N, et al. Screening for rheumatic heart disease: evaluation of a simplified echocardiography-based approach. Eur Heart J Cardiovasc Imaging. 2012;13: 1024-9.

29. Lu JC, Sable C, Ensing GJ, Webb C, Scheel J, Aliku T, et al. Simplified Rheumatic Heart Disease Screening Criteria for Handheld Echocardiography. J Am Soc Echocardiogr. 2015;28:463-9.

30. Mirabel M, Bacquelin R, Tafflet M, Robillard C, Huon B, Corsenac P, et al. Screening for rheumatic heart disease: evaluation of a focused cardiac ultrasound approach. Circ Cardiovasc Imaging. 2015;8.

31. Ploutz M, Lu JC, Scheel J, Webb C, Ensing GJ, Aliku T, et al. Handheld echocardiographic screening for rheumatic heart disease by non-experts. Heart. 2016;102:35-9.

32. Yoshida K, Yoshikawa J, Shakudo M, Akasaka T, Jyo Y, Takao S, et al. Color Doppler evaluation of valvular regurgitation in normal subjects. Circulation. 1988;78:840-7.

33. Webb RH, Gentles TL, Stirling JW, Lee M, O'Donnell C, Wilson NJ. Valvular Regurgitation Using Portable Echocardiography in a Healthy Student Population: Implications for Rheumatic Heart Disease Screening. J Am Soc Echocardiogr. 2015;28:981-8.

34. Webb RH, Wilson NJ, Lennon DR, Wilson EM, Nicholson RW, Gentles TL, et al. Optimising echocardiographic screening for rheumatic heart disease in New Zealand: not all valve disease is rheumatic. Cardiol Young. 2011;21: 436-43.

35. Roberts K, Colquhoun S, Steer A, Remenyi B, Carapetis J. Screening for rheumatic heart disease: current approaches and controversies. Nat Rev Cardiol. 2013;10:49-58.

36. Essop MR, Peters F. Contemporary issues in rheumatic fever and chronic rheumatic heart disease. Circulation. 2014;130:2181-8.

\section{Submit your next manuscript to BioMed Central and we will help you at every step:}

- We accept pre-submission inquiries

- Our selector tool helps you to find the most relevant journal

- We provide round the clock customer support

- Convenient online submission

- Thorough peer review

- Inclusion in PubMed and all major indexing services

- Maximum visibility for your research

Submit your manuscript at www.biomedcentral.com/submit
Biomed Central 